\title{
A Flexible Model for Moisture-Suction Relationship for Unsaturated Soils and Its Application
}

\author{
Nadarajah Ravichandran, Shada H. Krishnapillai \\ Civil Engineering Department, Clemson University, Clemson, USA \\ E-mail:nravic@clemson.edu \\ Received May 12, 2011; revised June 21, 2011; accepted July 25, 2011
}

\begin{abstract}
The mathematical equation for the moisture-suction relationship also known as soil water characteristic curve (SWCC) is one of the constitutive relations necessary for the computational modeling of deformation and flow problems of unsaturated soil using the finite element method. In this paper, a new empirical equation for the SWCC is developed that incorporates the actual air-entry suction and the maximum possible suction of the soil as input parameters. The capability of the new model is investigated by fitting the experimental data for twelve different soils that includes sands, silts, and clays. The model fits the experimental data well including in high suction range which is one of the difficulties observed in other commonly used models such as the Brooks and Corey, van Genuchten, and Fredlund and Xing models. The numerical stability and the performance of the new model at low and high degrees of saturations in finite element simulation are investigated by simulating the dynamic response of a compacted embankment and the results are compared with similar predictions made using widely used SWCC models.
\end{abstract}

Keywords: Soil-Water Characteristic Curve, Unsaturated Soils, SWCC for Low Degree of Saturation, Moisture-Suction Relationship, Comparison of Soil-Water Characteristic Curves

\section{Introduction}

In recent years, the importance of unsaturated soil mechanics and its applications in the design and constructions of safe and economical geotechnical engineering structures is realized by not only the academic researchers but also by practicing engineers. The slope failure after rainfall and the shrinking and swelling of clays are two of the many examples that require a better understanding of unsaturated soil mechanics principles. In contrast to saturated soil, variation in soil moisture content will have a great influence on the load bearing capacity, settlement and flow behavior of unsaturated soils. For example, a drop in moisture content will result in an increase in soil stiffness and strength, a decrease in soil compressibility and a decrease in water permeability. The increase/decrease in mechanical and flow behavior depends upon the type of soil. For example, clayey soil exhibits a greater change in stiffness and compressibility compared to sandy soils for the same moisture content change. From numerous observations, it is clear that the amount of water present in the soil, measured in any form such as degree of saturation or water content, has great influence on unsaturated soil behavior.

Unsaturated soil is a three phase porous media consisting of three bulk phases: solid skeleton, water, and pore air. In addition to these three bulk phases, there exist three interfaces: solid-water interface, solid-air interface, and water-air interface. Of the three, the water-air interface (contractile skin) that does not exist in either saturated or dry soil influences the unsaturated soil behavior (i.e., unsaturated soil behavior differs from saturated soil not only because of the presence of air phase but also by the presence of the water-air interface). The contractile skin maintains the pressure balance between water and air phases. The difference between the air pressure and water pressure is known as matric suction, which is a function of degree of saturation (amount of water) and other properties such as void ratio, void distribution, particle size distribution and initial density. The major difference between unsaturated and saturated soil mechanics is the influence of matric suction on its behavior, that is to say the mechanical and flow characteristics of unsaturated soil are affected by matric suction [1].

Numerical modeling of mechanical and flow problems 
in unsaturated soil requires a set of governing differential equations that represents the physics of the problem, a stress-strain relationship that relates the deformation of the soil body to the applied load and a moisture-suction relationship. The moisture-suction relationship not only affects the flow but also deformation characteristics of the soil body because suction is one of the two stress state variables widely used in the deformation analysis of unsaturated soils. These mathematical relationships must represent the true physics of the problem (material, boundary condition and loading) more closely for accurate predictions. It is obvious that mathematical models will become complex when they represent the true behavior more closely. The complexity can arise in the form of increased number of model parameters and/or complex formulations. The complex mathematical equations that perform well in single element test or small well defined problems may become numerically unstable when real world problems are simulated with realistic boundary and loading conditions. Also, when the number of model parameters increase, some of them may not have physical meaning and/or difficult to determine from simple laboratory results. This paper focuses on developing a flexible and numerically stable moisture-suction relationship for unsaturated soil.

The new empirical equation for the moisture-suction relationship presented in this paper seems capable of matching the measured data of various soils over a wide range of degree of saturation (near dry to fully saturated conditions). It can also be used either with residual water content (the lower bound value for the water potential) or with a maximum suction value (the upper bound value for the suction) at near dry conditions. The capability of the new model is verified by matching the experimental data of twelve soils that includes sands, silts, and clays. The performance and the stability of the proposed moisture-suction model in the finite element modeling of unsaturated soils is investigated by simulating the dynamic behavior of a compacted embankment subjected to earthquake shaking at a low degree of saturation.

\section{Review of Widely Used Soil-Water Characteristic Curves}

The amount of water present in the soil can be expressed in various forms such as degree of saturation (S), volumetric water content, or gravimetric water content. Also, the SWCC for a given soil can differ depending upon the wetting or drying process used to vary the moisture content in the soil sample. The portion of the SWCC obtained by the wetting a dry sample is called the primary wetting curve. Similarly, the curve obtained by drying a wet sample is called the primary drying curve. The pri- mary drying curve always exhibits higher suction compared to the wetting curve (Figure 1) for a given degree of saturation [2-9]. Although the wetting and drying curves differ significantly and show hysteretic loops, most of the mathematical models for the moisture-suction relationship are represented by a single equation [10-12] for easy use in finite element modeling.

\subsection{Factors Influencing the SWCC and Development Strategies}

Various mathematical models have been developed to fit the measured moisture-suction relationship of natural soils using empirical, statistical and microscopic procedures [10-16]. All of these models confirm an inverse proportional relationship between the degree of saturation and suction. This inverse relationship can be explained with the fundamental meniscus theory as follows. When the degree of saturation increases, the radius $\left(R_{s}\right)$ of the meniscus will also increase. When $R_{s}$ increases, the pressure difference between the pore air pressure and the pore water pressure (suction) will decrease as seen in Equation (1).

$$
\psi=\left(p^{\mathrm{g}}-p^{1}\right)=\frac{2 T_{s}}{R_{s}}
$$

where $\psi$ is the suction, $p^{g}$ is the pore air pressure, $p^{l}$ is the pore water pressure, and $T_{s}$ is the surface tension.

The air-entry suction and the pore size distribution index are two of the basic parameters incorporated in most of the widely used SWCC models and the effect of these two basic properties are represented by two parameters $\boldsymbol{a}$ and $\boldsymbol{n}$, respectively [10-12]. In addition to these two parameters, Kawai et al. [17] showed that the initial void ratio (e) affects the air-entry suction and proposed an inverse relationship between $\boldsymbol{a}$ and $\mathbf{e}$ as shown in Equation (2).

$$
a=160 \mathrm{e}^{-2.51}
$$

Another study by Vanapalli et al. [18] showed that the initial degree of saturation has significant influence on the shape of SWCCs at lower suction range. For example, a higher initial $\mathrm{S}$ makes the curves steeper, whereas the effect of initial $\mathrm{S}$ is insignificant at higher suction values. Through a series of experiments on undisturbed samples of completely decomposed volcanic soil with net normal stress levels of 0,40 and $80 \mathrm{kPa}, \mathrm{Ng}$ and Pang [2] showed the effect of net normal stress to be insignificant for some soils. Fredlund and Rahardjo [1] and Vanapalli et al. [18], studying the effect of total stress on the SWCCs, found that the air-entry suction parameter $\boldsymbol{a}$ increases with increasing equivalent pressure. The influence at high suction was investigated by Vanapalli et al. [18], which showed that the SWCC exhibits similar be 


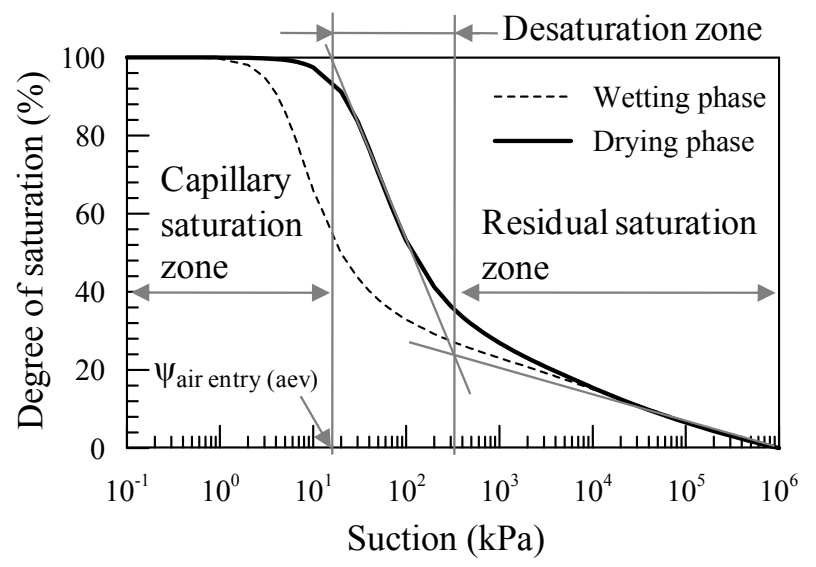

Figure 1. Typical SWCC with different regions of saturation.

haviors at high suctions $(20,000-300,000 \mathrm{kPa})$ even if all other parameters differ, i. e. effect of other factors being insignificant at high suction. Recent studies sows that the initial density of the soil also affects the SWCC [1]. Recent research publication shows that the uncertainty in the unsaturated soil properties should also be taken into account for accurate modeling of SWCC [19]. Although there are numerous SWCC models available in the literature, we have used the B-C [10], v-G [11] and F-X [12] models for further investigation before developing our new model and for comparing the applicability performance this model.

The B-C model shown in Equation (3) is one of the earliest models widely used in many applications including finite element codes. It is a non-smooth model consisting of two parts. An inverse power law is used beyond air-entry suction and 1.0 is used within the airentry suction for the effective degree of saturation. The sudden change at the air-entry value may cause numerical instabilities especially when taking derivatives of the curve as part of the development of general governing equations for the dynamics of unsaturated soils at low $\mathrm{S}$.

$$
S^{\mathrm{e}}=\left\{\begin{array}{ccc}
1 & \text { if } & \psi<a \\
(\psi / a)^{-n} & \text { if } & \psi>a
\end{array}\right.
$$

where $S^{\mathrm{e}}$ is the effective degree of saturation given by $S^{\mathrm{e}}=\left(S-S_{r}\right) /\left(1-S_{r}\right), \quad \psi$ is the suction and $\boldsymbol{n}$ is a fitting parameter related to the pore size distribution index of the soil, $S$ is the degree of saturation, $S_{r}$ is the residual saturation. Another widely used model is the $\mathrm{v}-\mathrm{G}$ model given in Equation (4) that provides a single smooth equation for the entire range of $\mathrm{S}$ introducing another fitting parameter $\boldsymbol{m}$.

$$
S^{\mathrm{e}}=\frac{1}{\left(1+(a \psi)^{n}\right)^{m}}
$$

where the fitting parameter $\boldsymbol{m}$ is related to the symmetry of the model that increases the flexibility in fitting experimental data of many different soils. The other widely used SWCC is the F-X model given in Equation (5) that incorporates a correction factor, $C(\psi)$. This correction factor forces the model to pass through a prescribed suction value of $10^{6} \mathrm{kPa}$ at near dry condition while the B-C and v-G models predict infinity that is considered unrealistic.

$$
\begin{array}{r}
S^{\mathrm{e}^{*}}=\frac{C(\psi)}{\left(\ln \left(\mathrm{e}+(\psi / a)^{n}\right)\right)^{m}} \\
C(\psi)=1-\frac{\ln \left(1+\frac{\psi}{C r}\right)}{\ln \left(1+\frac{1000000}{C r}\right)}
\end{array}
$$

where $S^{\mathrm{e}^{*}}$ is similar to $S^{\mathrm{e}}$ but $S_{r}$ is set to zero (used with maximum suction concept). The variable $e$ in Equation (5) is the natural logarithmic constant, and the parameter $C_{r}$ in the correction factor is a parameter related to the residual water content. Further investigation on the F-X model by Leong and Rahardjo [20] revealed that the correction factor $C(\psi)$ significantly affects the initial portion of the curve (capillary saturation, desaturation zones shown in Figure 1 when the $C_{r}$ is relatively low.

\subsection{Capabilities of the Widely Used Models and Model Parameter Calibration}

Our evaluation of the existing SWCC models is based upon 1) their capability to capture the moisture-suction relation over the entire range of degrees of saturation, especially in the low saturation ranges, and 2) their performance in a fully coupled finite element simulation of unsaturated soil. In addition to collecting information from previously published papers [20,21], the authors performed extensive studies on the influence of the model parameters in each of the widely used models. It should be noted here that the authors' intention is to use the model in a fully coupled finite element simulation of unsaturated soil. Therefore, the authors' opinion about the existing models may differ from others.

Although the elemental B-C model has two fitting parameters, it fails to fit the measured data in high suction range. This failure is due to the fact that the SWCC equation has no inflection point that is observed in many of the measured SWCCs. The absence of this inflection point limits its flexibility to match the experimental data. In the case of three-parameter models such as $\mathrm{v}-\mathrm{G}$ and F-X models, the fitting parameters are not independent of each other resulting in a random procedure to match the 
experimental data by adjusting one or more of these parameters. For example, $\boldsymbol{n}$ can be increased by increasing $\boldsymbol{a}$ and $\boldsymbol{m}$ simultaneously. When $\boldsymbol{m}$ increases, the slope of the curve will also slightly increase, resulting in the curve moving along the suction axis towards low suction values. The other disadvantage of these three-parameter models is the association of physical meaning to the parameter $\boldsymbol{a}$. Although it is referred to as the air-entry suction related parameter, $\boldsymbol{a}$ in these models is generally larger than the actual air-entry value of the soil. The value of $\boldsymbol{a}$ in these models falls within the desaturation zone, although the initial point of the desaturation zone is defined as the air-entry value. In the case of the F-X model, a selection of relatively smaller $C_{r}$ values also will affect the initial portion of the curve. Such influence will result in multiple combinations of model parameters that are undesirable for finite element application in which these parameters are coefficients of important terms when derivatives are calculated.

The moisture-suction relationship of unsaturated soils is observed by assuming either a reasonable lower bound value for the water potential (residual water content) or upper bound value for the suction (maximum suction). Though the F-X model uses a maximum suction of $10^{6}$ $\mathrm{kPa}$, it has no theoretical basis and is expected to vary from soil to soil. Although a maximum possible suction of $10^{6} \mathrm{kPa}$ is shown as the theoretical maximum suction [12], incorporating the maximum suction as part of the model will increase its flexibility in fitting the measured data well especially in low saturation ranges. In addition, researchers may wish to use both lower bound and upper bound concepts in a single numerical simulation with multiple unsaturated soil layers (i.e. in real boring logs for geotechnical engineering projects). For example, there might be a need to specify residual water content for clayey soils while preserving a maximum suction for sandy soils in a single simulation. Therefore, a model that can be used either with residual water content or maximum suction is desirable. Also the fitting parameters should be independent of each other so that a single set of parameters can be obtained from calibration. A realistic SWCC model must also include the actual airentry suction as a model parameter instead of a related parameter. In this paper, a new flexible SWCC model is presented that seems to have eliminated the shortcomings found in the widely used models and includes desirable features discussed above.

\section{Proposed Equation for Moisture-Suction Relationship (SWCC)}

The new model is developed to solve the aforementioned practical issues and to provide a SWCC model for use with either residual water content concept or maximum suction at near dry conditions. This new empirical equation is given in equation (6). The factors that influence a SWCC and the modelling strategies described in Section 2 were closely followed to develop the new equation. Also, one can understand by comparing the new and F-X models that the proposed model is similar to the F-X model in terms of the framework but differs significantly with new parameters and correction function.

$$
\begin{aligned}
& S^{\mathrm{e}^{*}}=\frac{N(\psi)}{\left(1+m \ln \left(1+\frac{1}{m}\left(\psi / a_{\text {air-entry }}\right)^{n}\right)\right)^{0.5}} \\
& N(\psi)=1-\left(\frac{N_{r}}{N_{r}-1+\psi_{\max } / \psi}\right)^{0.5}
\end{aligned}
$$

where $\boldsymbol{a}, \boldsymbol{n}, \boldsymbol{m}$, and $\boldsymbol{N}_{\boldsymbol{r}}$ are the fitting parameters; $\boldsymbol{\psi}_{\text {air-entry }}$ is the actual air-entry suction, $\boldsymbol{a}$ is a non-dimensional parameter that represents the ratio between the air-entry suction and the suction at inflection point in the curve. The parameter $\boldsymbol{n}$ is related to the pore-size distribution of the soil, and $\boldsymbol{m}$ is related to asymmetry of the model in moisture-suction plane similar to that of the $\mathrm{v}-\mathrm{G}$ and F-X models. $\psi$ is the suction, $\boldsymbol{\psi}_{\max }$ is the maximum suction or suction at near dry conditions, and $N_{r}$ is a number related to residual water content. Though the function $N(\psi)$ is similar to the function $C(\psi)$ in the F-X model, $N(\psi)$ does not affect the initial portion (portion in the low suction range) of the curve. The effect of $N(\psi)$ over a range of $N_{r}$ value (0 to 10) is discussed in the subsequent sections. The Equation (7) incorporating this residual water content $\left(\theta_{r}\right)$ is expressed below.

$$
\frac{\theta-\theta_{r}}{\theta_{s}-\theta_{r}}=\frac{1-\left(\frac{N_{r}}{N_{r}-1+\psi_{\max } / \psi}\right)^{0.5}}{\left(1+m \ln \left(1+\frac{1}{m}\left(\psi / \text { a } \psi_{\text {air-entry }}\right)^{n}\right)\right)^{0.5}}
$$

If the maximum suction concept is to be considered, the Equation (7) can be simply reduced by setting $\theta_{r}$ to zero $\left(\theta_{r}=0\right)$ together with a maximum suction $\left(\psi_{\max }\right)$ and a calibrated $N_{r}$ value. Conversely, if residual water content of the soil has to be considered, the factor $N_{r}$ can be set at zero $\left(N_{r}=0\right)$. When $N_{r}$ is equal to zero, the function $N(\psi)$ will be unity thusly reducing it to an Equation (8) that can handle the residual water content concept.

$$
\frac{\theta-\theta_{r}}{\theta_{s}-\theta_{r}}=\frac{1}{\left(1+m \ln \left(1+\frac{1}{m}\left(\psi / a \psi_{\text {air-entry }}\right)^{n}\right)\right)^{0.5}}
$$




\subsection{Derivatives of the New SWCC Model}

One of the instances in which the derivative of the moisture-suction relation is required in the finite element simulation of unsaturated soil is in the mass balance equation for the water and air phases. The necessary derivatives of the new model are given below in Equations (9) and (10).

$$
\frac{\mathrm{d} \theta}{\mathrm{d} \psi}=\left(\theta_{s}-\theta_{r}\right) \frac{\mathrm{d} S}{\mathrm{~d} \psi}
$$

\subsection{Fitting Parameters in the New Model}

Understanding the role of each parameter in the analytical model is important for adjusting these parameters to fit the experimental data well to obtain the best set of parameters. A detailed discussion based on the parametric studies performed upon the role of each parameter is presented below.

\subsubsection{Role of Parameter $a$}

The parameter $\boldsymbol{a}$ is the ratio between the actual air-entry suction of the soil and the suction at the inflection point of the SWCC. The effect of parameter $\boldsymbol{a}$ on the shape of the SWCC in the proposed model is shown in Figure 2. In the case of B-C, V-G and F-X models, the curve can be shifted along the suction axis by increasing $\boldsymbol{a}$. However, in the proposed model, $\boldsymbol{a}$ must be first adjusted until the initiation point of the desaturation zone (Figure 1) matches the air-entry suction ( $\left.\psi_{\text {air-entry }}\right)$ of the soil. For sandy soils, since the slope of the curve is steeper, the value of $\boldsymbol{a}$ will be relatively small. From authors experience, $\boldsymbol{a}$ ranges between zero and two. For clayey soils the slope is mild and the value of $\boldsymbol{a}$ is higher than five. The value of $\boldsymbol{a}$ for silty soils, generally, falls between that of clay and sand (approximately between 1 and 5).

\subsubsection{Role of Parameter $n$}

The influence of the parameter $\boldsymbol{n}$ in the shape of the SWCC is shown in Figure 3. As seen here, $\boldsymbol{n}$ changes the slope of the curve about the inflection point. When $\boldsymbol{n}$

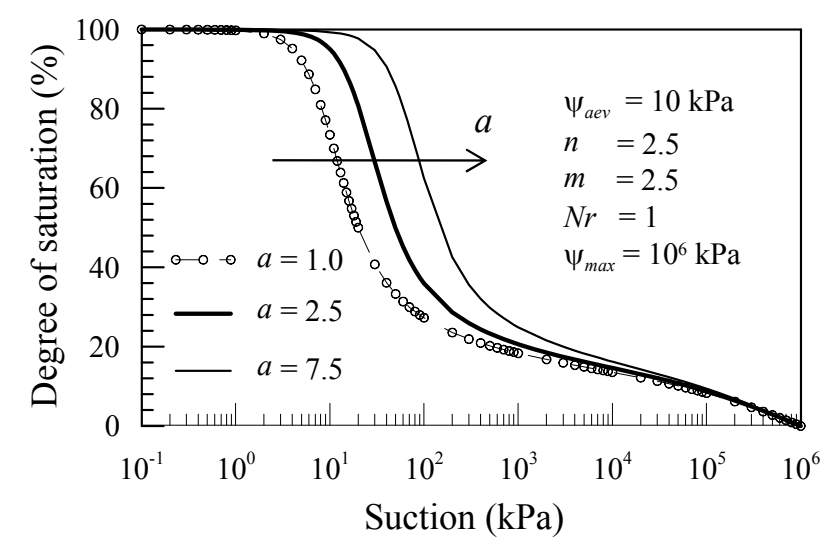

Figure 2. Influence of the parameter $a$ in the new SWCC model.

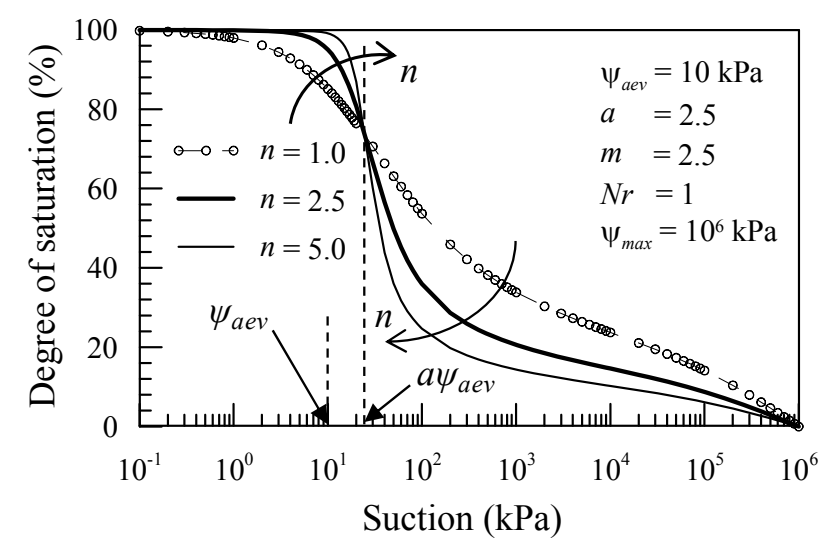

Figure 3. Influence of the parameter $n$ in the new SWCC model.

increases the Sat a given suction will increase if the suction is greater than $a \psi_{\text {air-entry }}$, and the Swill decrease if the suction is less a $\psi_{\text {air-entry. }}$.

\subsubsection{Role of Parameter $m$}

Influence of $\boldsymbol{m}$ in the shape of the SWCC is shown in Figure 4(a). As seen here, $\boldsymbol{m}$ in the proposed model does not affect the curve when the suction is within $\mathbf{0}$ and $\boldsymbol{a} \boldsymbol{\psi}_{\text {air-entry. }}$. This indicates that the parameter $\boldsymbol{m}$ does not alter the shape of the curve that may require re-adjustment of the parameters $\boldsymbol{a}$ and $\boldsymbol{n}$. On the other hand, the

$$
\begin{aligned}
\frac{\mathrm{d} S}{\mathrm{~d} \psi}= & \frac{-1}{\left(1+m \ln \left(1+\frac{1}{m}\left(\psi / a \psi_{\text {air-entry }}\right)^{n}\right)\right)^{m}} \\
& \times\left(\frac{d N(\psi)}{d \psi}+\frac{n\left(\psi / a \psi_{\text {air-entry }}\right)^{n} N(\psi)}{2 \psi\left(1+\frac{1}{m}\left(\psi / a \psi_{\text {air-entry }}\right)^{n}\right)\left(1+m \ln \left(1+\frac{1}{m}\left(\psi / a \psi_{\text {air-entry }}\right)^{n}\right)\right)}\right)
\end{aligned}
$$


parameter $\boldsymbol{m}$ in the $\mathrm{v}-\mathrm{G}$ and F-X models alters the slope of the curve and the predicted air-entry suction as seen in Figure 4(b). This requires readjustment of $\boldsymbol{a}$ and $\boldsymbol{n}$ to fit the experimental curve. This not only requires a tedious calibration procedure, but results in multiple possible combinations of model parameters and difference in finite element simulation results for the same soil.

\subsubsection{Role of Parameter $\mathrm{Nr}$ and $\psi_{\max }$}

The influence of the parameter $\boldsymbol{N}_{r}$, a parameter in the correction factor in the new model, in reaching the specified maximum suction is shown in Figure 5. As mentioned previously, one of the advantages of the proposed model is the use of maximum suction as a model parameter in which maximum suction must be obtained from experimental results and used in the modeling. In the example shown in Figure 5, the maximum suction of $10^{6} \mathrm{kPa}$, a theoretical value obtained based on thermodynamic principles for any soil [4], is used. As seen there, because the parameter $\mathrm{N}_{\mathrm{r}}$ does not affect the initial portion (capillary saturation, desaturation zones) of the curve, the effect of $\mathrm{Nr}$ on the other model parameters is insignificant.

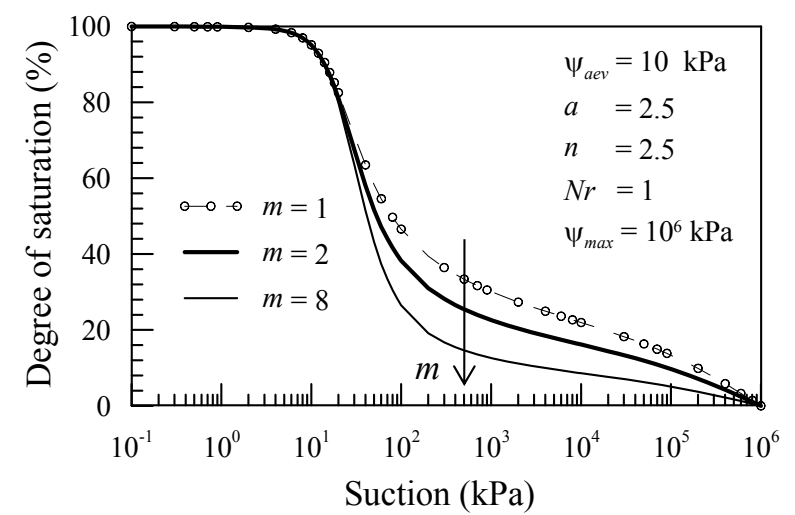

(a)

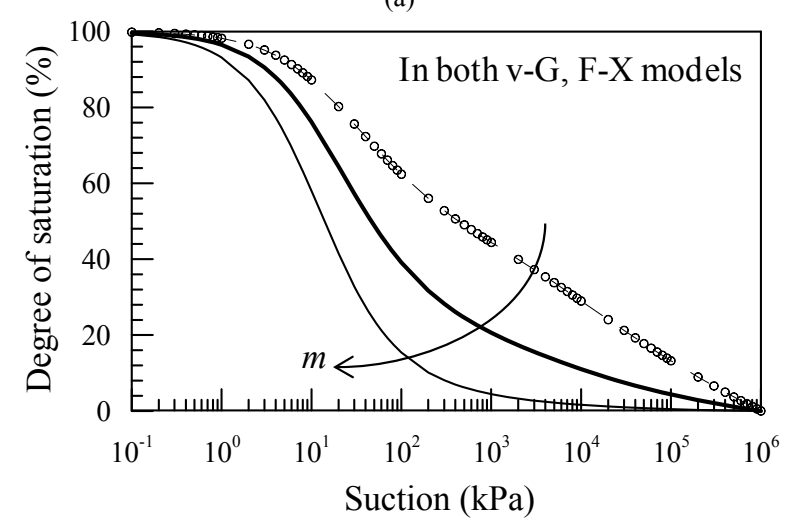

(b)

Figure 4. Influence of the $m$ in the new and two other widely used SWCC models.

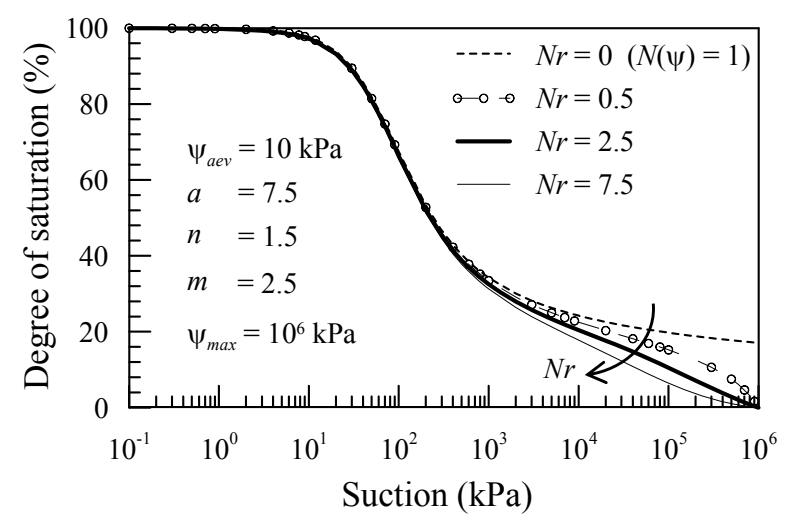

Figure 5. Influence of the $N r$ in the new SWCC model.

As shown in Equation (8), the correction factor $N(\psi)$ must be 1.0 to use the residual water content concept. Setting $N_{r}$ equals to zero (given that $\psi$ is not equal to $\psi_{\max }$ ) can yield this correction factor. Based upon our experience, $N_{r}$ varies within 1.0 and 5.0 for any soil. Figure 6 shows the use of $\psi_{\max }$.

\section{Predictive Capability of the New Model}

The capability of the new model in predicting the moisture-suction relation for twelve different soils that include sands, silt and clays is investigated. Due to limitations in page number, comparisons for six soils are presented in this paper. The calibrated SWCCs together with the model parameters are presented in Figure 7, in which the $\psi_{\text {air-entry }}$ is denoted as $\psi_{\text {air }}$. The predicted SWCC without the correction factor $N(\psi)$ is also presented for comparison purposes. Figures 7(a) and (b) show the calibration of SWCC model for a Superstition sand (data [22]) and Lakeland sand (data - [23]), respectively. Figures 7(c) and (d) show the calibration of SWCC model parameters for Touchet silt loam (data - [10]) and Botkin silt (data - [18]), respectively. Figures 7(e) and (f) show the calibration of SWCC model parameters for

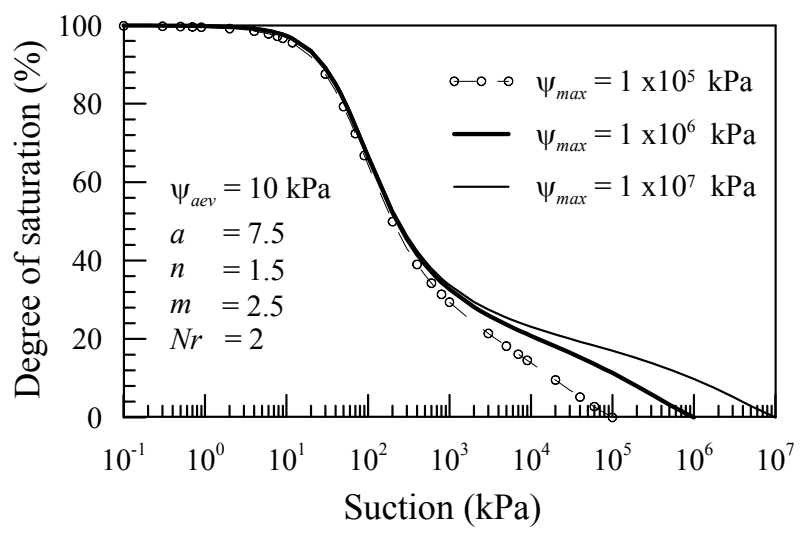

Figure 6. Effectiveness of the $\psi_{\max }$ in the new SWCC model. 

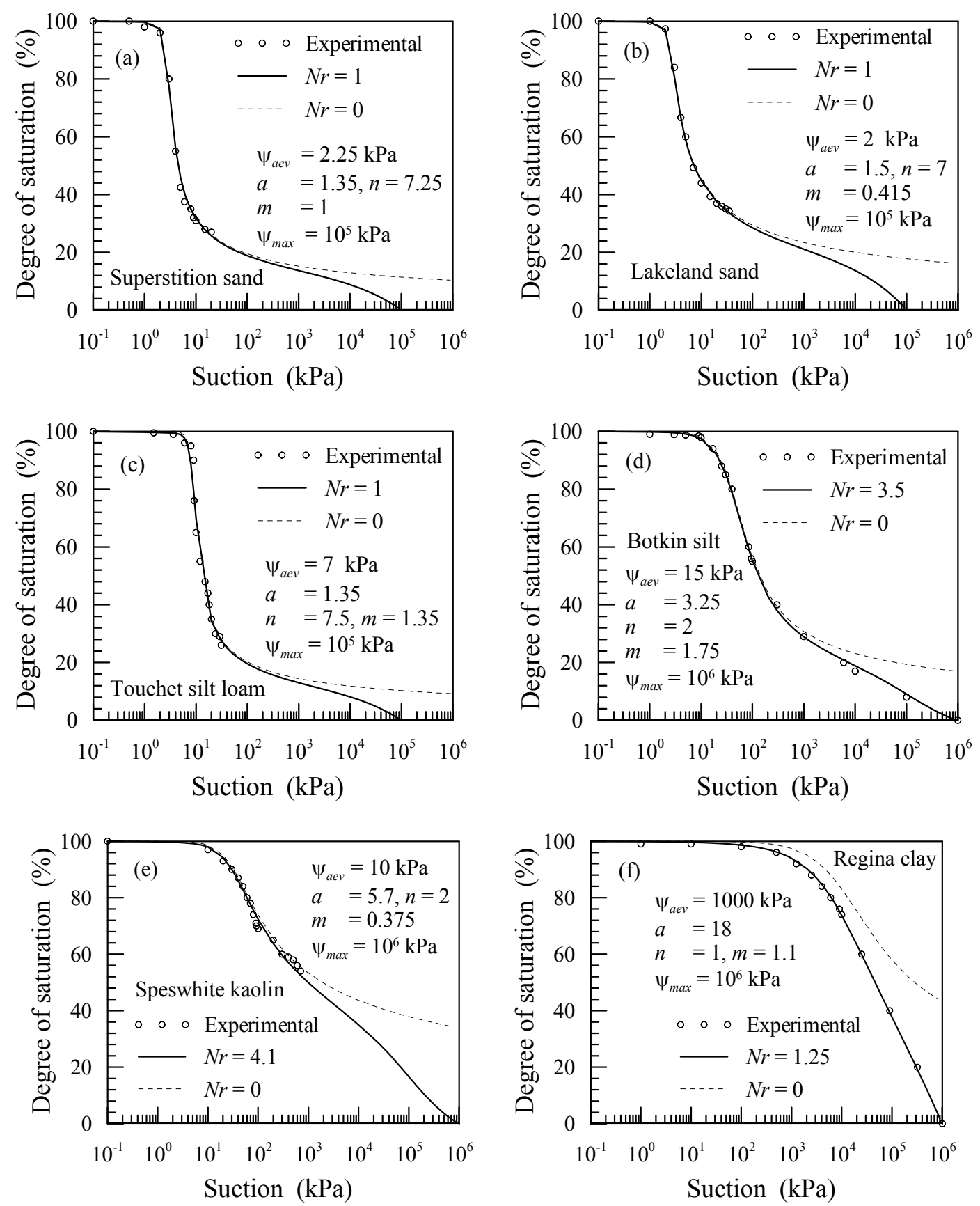

Figure 7. Calibration of model parameters for various soils for both residual water content and maximum suction concepts.

Speswhite kaolin (data [24]) and Regina clay (data [18]), respectively. It should be noted that the experimental moisture-suction data for these soils are unavailable for the full degree range of saturation $(0-100 \%)$. The best estimates for both the air-entry suction $\left(\psi_{\text {air-entry }}\right)$ and maximum suction $\left(\psi_{\max }\right)$ for each soil are selected based upon the variation of available experimental data.

These results show that the new model is effective and flexible enough to fit the experimental data. The number $N_{r}$ in the new model can be chosen between 1 and 5 for any soil and the correction factor $N(\psi)$ does not affect the initial portion of the curve. The new model can be effectively used with either the residual water content concept or with a maximum suction value at near dry conditions. In addition to eliminating this deficiency, the new model includes all the advantages of the B-C, $\mathrm{v}-\mathrm{G}$ and F-X models. The performance of the new SWCC model in finite element simulations is investigated by simulating a compacted embankment at various initial degrees of saturation subjected to earthquake shaking. 


\section{Performance of the New SWCC in Finite Element Simulations}

One of the primary applications of mathematical representation of soil-moisture relation in geotechnical engineering is in the finite element modelling of unsaturated soils subjected to various loadings and boundary conditions. In general, mathematical equations become complex when they represent the true behaviour while the complex equations simultaneously limit the application of such equations in boundary value problems via numerical instabilities. This statement is true for governing differential equations that are spatially discretized to form finite element equations, stress-strain relationship and moisture-suction relationship. Because of the fact that different elements in a problem domain (finite element mesh) can be at different levels of degrees of saturation when subjected to flux and traction boundary conditions, a single moisture-suction model should have the flexibility and stability to be used in finite element simulations.

\subsection{Finite Element Simulation Tool for Unsaturated Soils}

Although complete and partially reduced finite element formulations are available [25], a simplified finite element formulation for unsaturated soil [24] is used in this study. The simplified formulation is free of numerical instabilities arise from other sources for the problems analyzed in this paper [25] and can easily capture if there is any numerical instabilities occur due to proposed SWCC. The complete governing differential equations representing the physics of unsaturated soil derived using mass balance, momentum balance and laws of thermodynamics was simplified by neglecting the relative accelerations and velocities of the pore air and water phases. The corresponding finite element equations are solved considering the solid displacement as the primary nodal unknowns, and the element fields (e.g. water pressure and air pressure) are calculated using the mass balance equations. This simplified formulation represents the undrained soil condition since the relative accelerations and velocities are neglected. Therefore, the change in degree of saturation in a finite element occurs due to the deformation (volumetric deformation) of the solid skeleton (a finite element). When the degree of saturation changes due to deformation, corresponding matric suction is calculated using the SWCC. The reason for selecting the reduced formulation is to isolate the numerical instabilities arising from other sources such as governing equations (as in the case of complete formulation). Also, because the permeability coefficient of water in unsaturated state is much smaller than in saturated soil states, we may assume that unsaturated soils behave in an undrained condition especially under earthquake shaking.

\subsection{Simulation Results and Discussion}

To show the influence of various SWCC models, the dynamic behavior of a compacted earthen embankment made of Speswhite Kaolin subjected to earthquake shaking at the base was simulated using the finite element code described previously. The finite element mesh shown in Figure 8 consists of 292 quadrilateral elements. The base of the embankment was assumed to be impermeable and fixed in all directions throughout the analysis, whereas all other sides of the embankment were assumed to be traction free. The embankment was numerically shaken with the acceleration time history shown in Figure 9, and the stress-strain relationship of the soil was assumed to be linear elastic. Again, the reason for selecting the simplest stress-strain relationship is to isolate the numerical instabilities arising from the complex elasto-plastic constitutive model. The linear elastic model parameters and other soil properties are shown in Table 1.

Simulations were performed using all four models, B-C, v-G, F-X and our proposed model (S-R). The SWCC model parameters were calibrated against the experimental data published by Sivakumar [23]. The

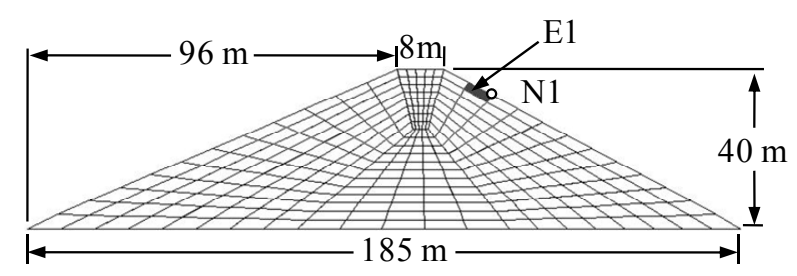

Figure 8. Finite element mesh of the compacted embankment.

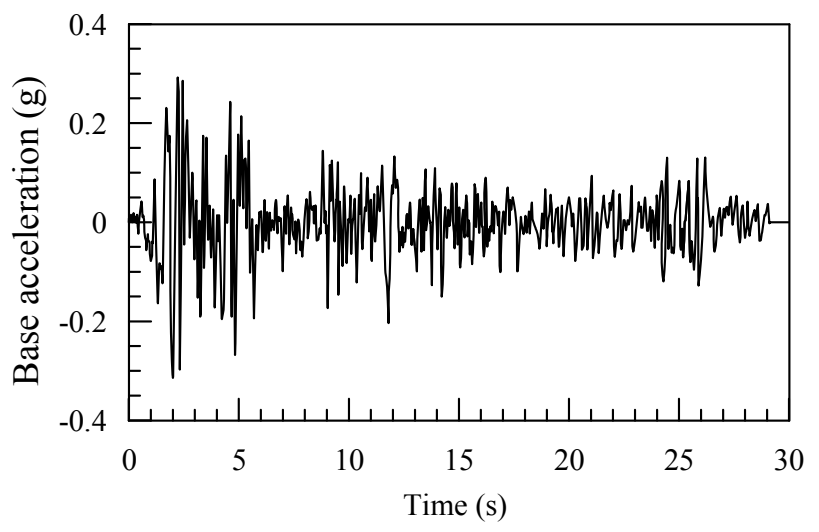

Figure 9. Base acceleration time history. 
Table 1. Linear elastic model parameters for Speswhite kaolin.

\begin{tabular}{lcc}
\hline \multicolumn{2}{c}{ Properties } & Value \\
\hline Solid grain density & $\mathrm{Mg} / \mathrm{m}^{3}$ & 2.62 \\
Liquid density $\quad \mathrm{Mg} / \mathrm{m}^{3}$ & 1.0 \\
Gas density $\left(\times 10^{-3}\right)$ & $\mathrm{Mg} / \mathrm{m}^{3}$ & 2.1 \\
Bulk modulus of liquid $\left(\times 10^{6}\right) \mathrm{kPa}$ & 2.2 \\
Bulk modulus of gas $\quad \mathrm{kPa}$ & 101.3 \\
Viscosity of liquid $\left(\times 10^{-6}\right) \quad \mathrm{kPa} \cdot \mathrm{s}$ & 1.0 \\
Viscosity of gas $\left(\times 10^{-8}\right) \quad \mathrm{kPa} \cdot \mathrm{s}$ & 1.0 \\
Young's Modulus $\left(\times 10^{5}\right) \mathrm{kPa}$ & 0.3 \\
Poisson's ratio & 0.2 \\
\hline
\end{tabular}

calibrated model parameters for the B-C model are: $\boldsymbol{a}=$ $17 \mathrm{kPa}, \boldsymbol{n}=0.18$, irreducible $\mathrm{S}=0$; for the $\mathrm{v}-\mathrm{G}$ model: $\boldsymbol{a}$ $=0.058 \mathrm{kPa}-1, \boldsymbol{n}=2.85, \boldsymbol{m}=0.063$, irreducible $\mathrm{S}=0$; for the F-X model: $\boldsymbol{a}=28 \mathrm{kPa}, \boldsymbol{n}=1.65, \boldsymbol{m}=0.365, C r=$ $5,000 \mathrm{kPa}$; for the new model: $\psi_{\text {air-entry }}=10 \mathrm{kPa}, \boldsymbol{a}=5.7$, $\boldsymbol{n}=2, \boldsymbol{m}=0.375, N r=4.1$, and $\psi_{\max }=10^{6} \mathrm{kPa}$. In the new model, the air-entry suction $\left(\psi_{\text {air-entry }}\right)$ and the maximum suction $\left(\psi_{\max }\right)$ values were selected by looking at the trend of the experimental curve.

As mentioned previously, all the models predict identical responses when the degree of saturation falls in the mid-range and show significant differences or become inapplicable in low and/or high degree of saturation range. Therefore, in this paper, the initial degree of saturations of $10 \%, 25 \%$ and $90 \%$ were selected for the finite element simulations. The $\mathrm{S}$ corresponding to the residual water content (irreducible S) is set to be zero in the B-C and $\mathrm{v}-\mathrm{G}$ models to simulate identical soil condition in all four models. The predicted incremental matric suction time histories in element E1 (Figure 8) are presented in Figure 10. As shown in the figures, while the initial $S$ increases the initial suction and the suction variation due to external loading decrease. The comparison study shows that the results predicted using the v-G, F-X models are close for an initial S of $90 \%$, the B-C model predicts a slightly lower suction variation as shown in Figure 10(b). As seen in Figure 10(a), significant differences are observed when the initial degree of saturation is $10 \%$. At $10 \%$ initial $\mathrm{S}$, the new and the F-X models also predict close responses, but the other two models could not be used in this analysis. The accuracy of the predicted response could not be verified due to the lack of experimental results for the finite element problem shown. However, this finite element simulation study shows that the new model is numerically stable and can be effectively used to capture the moisture-suction variation with wide range of initial S, especially at low degrees of saturation.
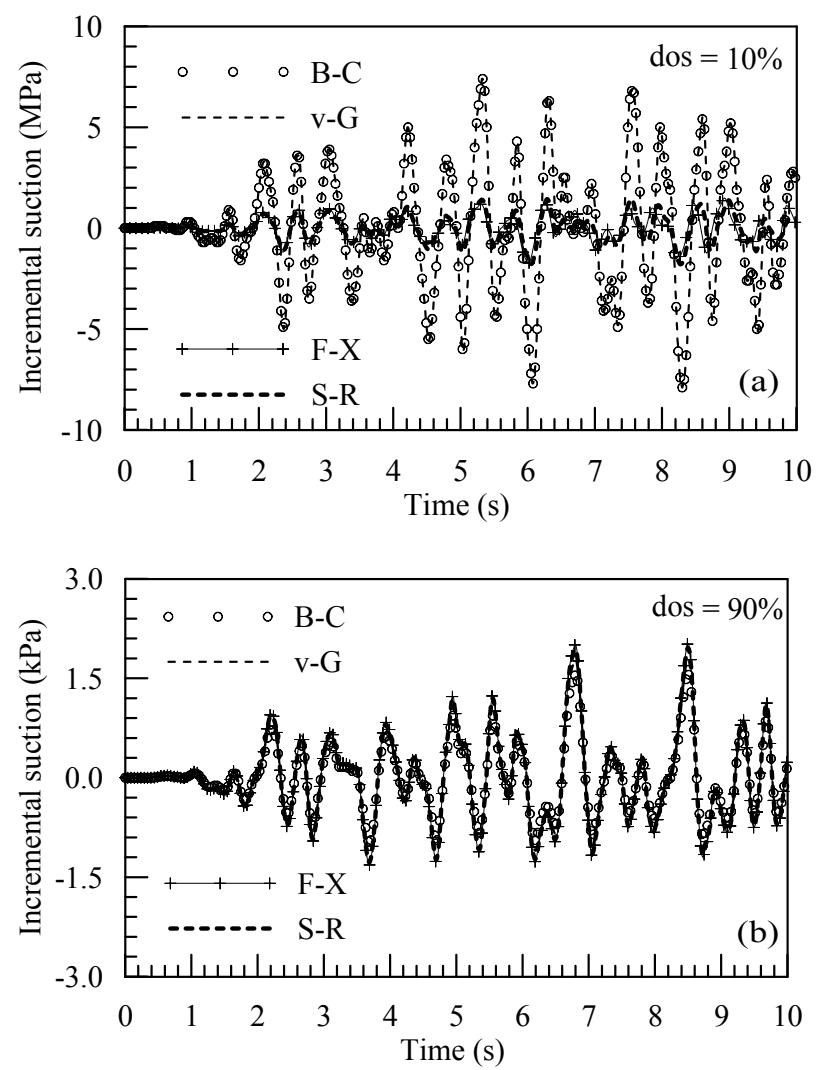

Figure 10. Predicted suction variation with different initial dos for the dynamic analysis of the embankment (dos-degree of saturation).

\section{Conclusions}

The proposed empirical equation for moisture-suction relationship in unsaturated soil seems flexible enough to match the experimental data at low degrees of saturation and numerically stable in finite element simulations of dynamic problems. The new model can be used either with a residual water content (lower bound value for the water potential) or with a maximum suction value (upper bound value) for dry case. If the maximum suction and air entry suctions are available for a soil, this data can be directly used in the proposed model. The performance of the new model is verified by fitting the experimental data of twelve different soils. The calibration results show that the new model can be successfully used to model various types of soils over a wide range of degree of saturation without any numerical difficulties. The factor $N(\psi)$ that is introduced to ensure the above capability is effective and numerically stable. Unlike the range of correction factor, $\boldsymbol{C}_{\boldsymbol{r}}$, in the F-X, the correction factor, $\left(\boldsymbol{N}_{r}\right)$, in the new model has a small range (it is recommended to use 1-10). The initial portion of the SWCC is not affected by the correction factor $N(\psi)$ as opposed to F-X model and the $N(\psi)$ enables the control of high 
suction portion independently.

\section{References}

[1] D. G. Fredlund and H. Rahardjo, "Soil Mechanics for Unsaturated Soils. A Wiley-Interscience Publication," John Wiley and Sons, Hoboken, 1993.

[2] C.W.W. Ng and Y.W. Pang, "Influence of Stress State on Soil-Water Characteristics and Slope Stability," Journal of Geotechnical and Geoenvironmental Engineering, Vol. 126, No. 2, 2000, pp. 157-166. doi:10.1061/(ASCE)1090-0241(2000)126:2(157)

[3] C. E. Zapata, "Uncertainty in Soil-Water Characteristic Curve and Impact on Unsaturated Shear Strength Predictions," Ph.D. Dissertation, Arizona State University, Tempe, 1999.

[4] Y. Mualem, "Extension of the Similarity Hypothesis Used for Modeling the Soil Water Characteristics," Water Resources Research, Vol. 13, No. 4, 1977, pp. 773-780. doi:10.1029/WR013i004p00773

[5] D. B. Jaynes, "Comparison of Soil-Water Hysteresis Models," Journal of Hydrology, Vol. 75, No. 1-4, 1985, pp. 287-299. doi:10.1016/0022-1694(84)90054-4

[6] W. L. Hogarth, J. Hopmans, J. Y. Parlange and R. Haverkamp, "Application of a Simple Soil-Water Hysteresis Model," Journal of Hydrology, Vol. 98, No. 1-2, 1988, pp. 21-29. doi:10.1016/0022-1694(88)90203-X

[7] J. R. Nimmo, "Semi-Empirical Model of Soil Water Hysteresis," Soil Science Society of America Journal, Vol. 56, No. 6, 1992, pp. 1723-1730. doi:10.2136/sssaj1992.03615995005600060011x

[8] Q. H. Pham, D. G. Fredlund and S. L. Barbour, "A Practical Hysteresis Model for the Soil-Water Characteristic Curve for Soils with Negligible Volume Change," Géotechnique, Vol. 53, No. 2, 2003, pp. 293-298.

[9] H. Yang, H. Rahardjo, E. Leong, C. and D. G. Fredlund, "Factors Affecting Drying and Wetting Soil-Water Characteristic Curves of Sandy Soils," NRC Research Press, Montreal, 2004.

[10] R. H. Brooks and A. T. Corey, "Hydraulic properties of porous media. Hydrology Paper, Colorado State University," Fort Collins, Vol. 27, No. 3, 1964.

[11] M. Th. van Genuchten, "A Closed-Form Equation for Predicting the Hydraulic Conductivity of Unsaturated Soils," Soil Science Society of America Journal, Vol. 44, No. 5, 1980, pp. 892-898. doi:10.2136/sssaj1980.03615995004400050002x

[12] D. G. Fredlund and A. Xing, "Equations for the Soil-Water Characteristic Curve," Canadian Geotechnical Journal, Vol. 31, No. 4, 1994, pp. 521-532. doi:10.1139/t94-061

[13] W. Gardner, "Mathematics of Isothermal Water Conduction in Unsaturated Soils," Highway Research Board Special Report 40, International Symposuim on PhysicoChemical Phenomenon in Soils, Washington, 1956, pp.
78-87.

[14] W. Brutsaert, "Probability Laws for Pore Size Distributions," Soil Science, Vol. 101, No. 2, 1966, pp. 85-92. doi:10.1097/00010694-196602000-00002

[15] C. McKee and A. Bumb, "Flow-Testing Coalbed Methane Production Wells in the Presence of Water and Gas," SPE Formation Evaluation, Vol. 2, No. 4, 1987, pp. 599-608.

[16] K. Kosugi, "The Parameter Lognormal Distribution Model for Soil Water Retention," Water Resource Research, Vol. 30, No. 4, 1994, pp. 891-901. doi:10.1029/93WR02931

[17] K. Kawai, D. Karube and S. Kato, "The Model of Water Retention Curve Considering Effects of Void Ratio" In: H. Rahardjo, D. G. Toll and E. C.Leong, Eds., Unsaturated Soils for Asia, Balkema, Rotterdam, 2000, pp. 329-334.

[18] S. K. Vanapalli, D. E. Pufahl and D. G. Fredlund, "The Influence of Soil Structure and Stress History on the Soil-Water Characteristic of a Compacted Till," Geotechnique, Vol. 49, No. 2, 1999, pp. 143-159. doi:10.1680/geot.1999.49.2.143

[19] H. B. Dye, S. L. Houston and B. D. Welfert, "Influence of Unsaturated Soil Properties Uncertainty on Moisture Flow Modeling," Geotechnical and Geological Engineering, Vol. 29, No. 2, 2011, pp. 161-169.

[20] E. C. Leong and H. Rahardjo, "Review of Soil-Water Characteristic Curve Equations," Journal of Geotechnical and Geoenvironmental Engineering, Vol. 123, 1997, pp. 1106-1117. doi:10.1061/(ASCE)1090-0241(1997)123:12(1106)

[21] W. S. Sillers, D. G. Fredlund and N. Zakerzadeh, "Mathematical Attributes of Some Soil-Water Characteristic Curve Models," Geotechnical and Geological Engineering, Vol. 19, No. 3-4, 2001, pp. 243-283. doi:10.1023/A:1013109728218

[22] L. A. Richards, "Water Conducting and Retaining Properties of Soils in Relation to Irrigationm" Proceedings, International Symposium on Desert Research, Jerusalem. 1952, pp. 523-546.

[23] A. Elzeftawy and K. Cartwright, "Evaluating the Saturated and Unsaturated Hydraulic Conductivity of Soils," In: T. F. Zimmie and C. O. Riggs, Eds., Permeability and Groundwater Contaminant Transport ASTM STP 746, ASTM, West Conshohocken, 1981, pp. 168-181. doi:10.1520/STP28323S

[24] V. Sivakumar, "A Critical State Framework for Unsaturated Soil," Ph.D. Thesis, University of Sheffield, Sheffield, 1993.

[25] N. Ravichandran and K. K. Muraleetharan, "Dynamics of Unsaturated Soils Using Various Finite Element Formulations," International Journal for Numerical and Analytical Methods in Geomechanics, Vol. 33, No. 5, 2009, pp. 611-631. doi:10.1002/nag.737 